\title{
Suzie's Dance: Reliving Dance through Motion Capture Animation of Photographs
}

\author{
Marco Gillies, Shakti Zapata Gomez and Sebastian ZImmer \\ Goldsmiths' University of London \\ New Cross, London SE14 6NW, UK \\ m.gillies@gold.ac.uk
}

\section{INTRODUCTION}

'Suzie's Dance' is a character driven documentary that explores the passions, life-force and sheer determination of a dancer who, suddenly cut down by a debilitating physical condition that led her in a wheelchair for the rest of her life, continues to follow her dreams, while guiding others to believe in, practice and develop their owns one.

This paper will describe the development of a section of the documentary that attempts reconstruct the dancer's childhood and youth while she was dancing without using a wheelchair and when discussing her childhood and youth. No video footage exists of her dancing at this time only photographs. We worked to bring these photographs alive as an animation story $/$ dance piece. This was done by motion capturing a performance by an able bodied dancer and applying it to these photographs so as to make them move. This is an important aesthetical element to cover the lack of footage in her past, and also an evocation of how memories and time can be constructed and can have an extended reality.

This work raises important challenges that are both technological and artistic. It attempts to recreate dance, at art form that is innately dynamic, threedimensional and corporeal from photographs which are static and two-dimensional. As such we have used a tight technico-artistic collaboration in which both technology and choreography must each adapt to the constraints of the other. While technology alone cannot solve the problem a flexible interaction of artistic decisions and software development can produce a workable, bespoke solution.

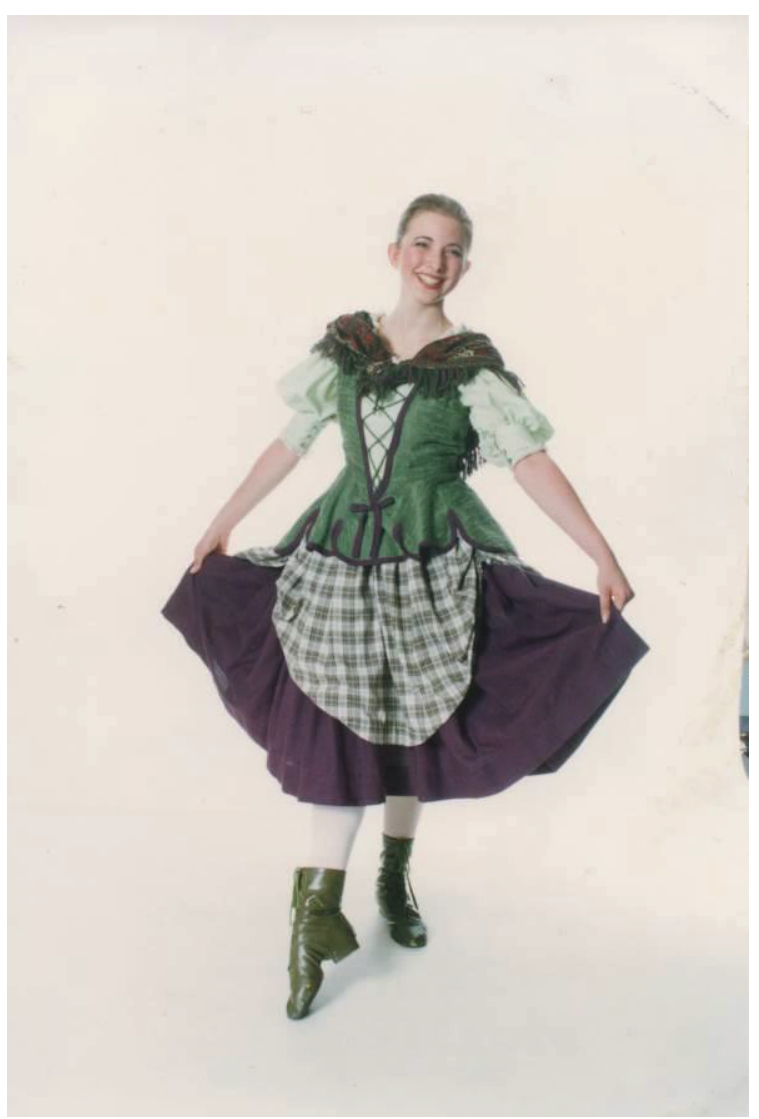

Figure 1: Suzie in her youth

\section{TECHNICAL CHALLENGES}

We have adapted the technique of smooth skinning which is used for motion capture animation of 3D characters to work with $2 \mathrm{D}$ photographs. This involves mapping a representation of a human skeleton onto the photograph as shown in figure 2 . The skeleton representation consists of rotational joints linked by fixed length bones. This skeleton is controlled via motion capture data which then animates the photograph. 


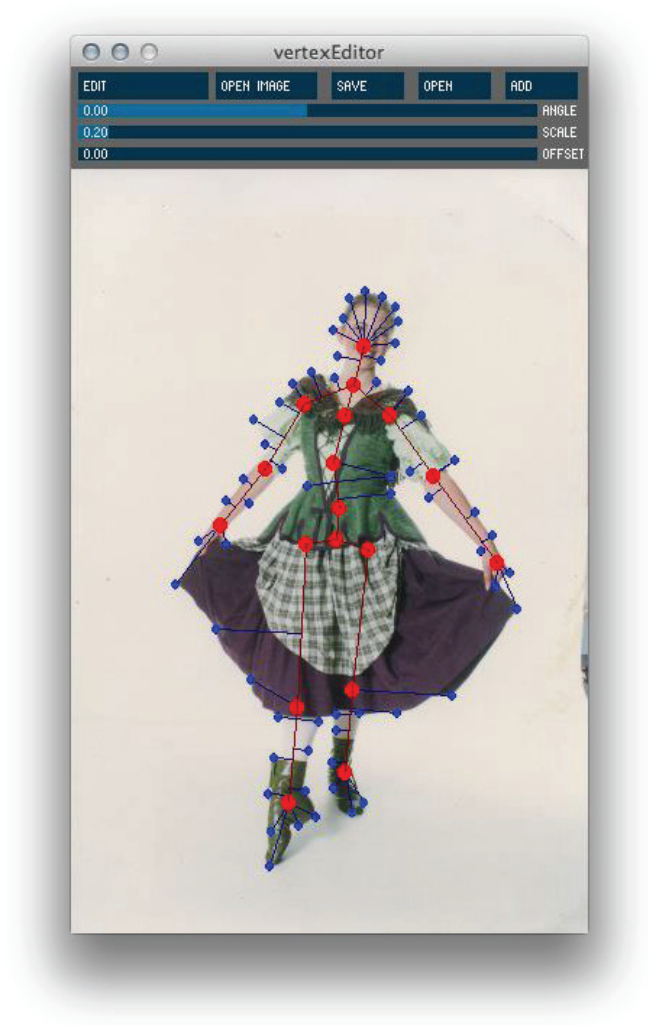

Figure 2: Our application: mapping a skeleton to a photograph

In order to perform this mapping we need to provide correspondences between the skeleton and the photograph. This is achieved by selecting point on the edge of the body that will map to the skeleton. These points are linked to one or more bones, which will control the movements of the points as the skeleton moves.

The points are formed into triangles and these triangles are texture mapped with elements of the original photograph (Figure 3 left).

Once this is done the skeleton representation can be animated using motion capture data. The points on the body are moved based on a weighted sum of the movements of the skeleton points they are attached to. The movements of the points results in deformations of the triangles, which in turn results in transformations of the images texture mapped to those triangles. This gives the appearances of the photograph moving in a way that follows the motion capture data.

The data was driven by motion capture data from a NaturalPoint Optitracktm optical motion capture system and a Microsoft Kinecttm markerless motion capture system.
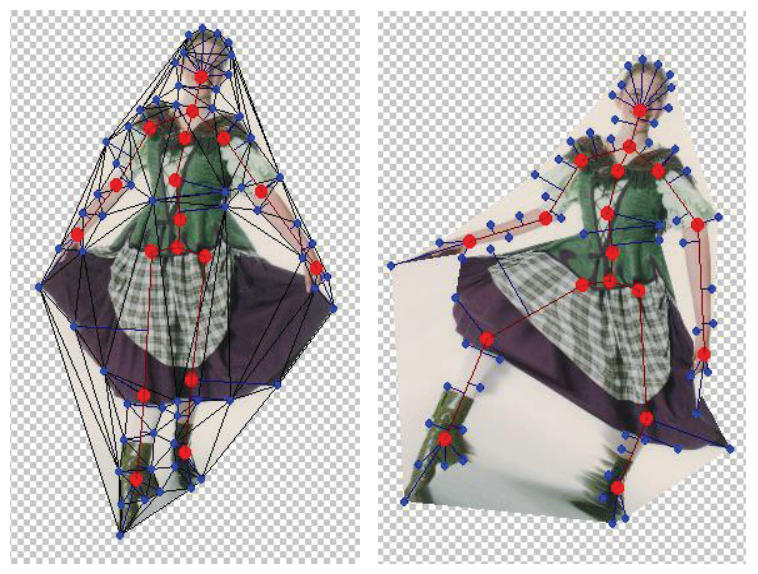

Figure 3: Left: triangulating the vertices. Right: moving the skeleton

\section{ARTISTIC CHALLENGES}

The method just described allows the photographs to move based on motion capture data of a dancers movements, but still leaves many open questions as the data is mapped from three to two dimensions. Data can be lost or distorted as movements that go outside the flat plane of the photograph must be mapped to an image that only exists in that plane. This requires solutions that are both technological and artistic as non-linear mappings and careful choreography are used to create an animation that is as close as possible to the dancer's technique while remaining faithful to the original photographic material. We use live motion capture improvisation and a tight loop of technology development driven closely by choreographic aims to create a new approach to motion capture performance, one that can reveal important lessons about performance, photography and technology.

\section{CONCLUSIONS}

This paper illustrates a challenge in the rerepresentation of dance using vintage photographs. What is interesting about this challenge is that at a purely technical level it is not solvable. There is simply too little information in a 2 dimensional photograph to reproduce full three dimensional movement. The movements of a dancer are too complex to map successfully on to photographs in a general way. However, the problem can be solved in a technico-artistic way, in which the technology can be developed to best match the choreographic intent and the choreography adapts to work within the constraints of what is possible technologically. 